\title{
Histochemical Patterns of Dehydrogenase Activity in the Development of Free Muscle Grafts in the Rat
}

\author{
DAVINDRA K. MAGON, MARC D. BASSON, AND BRUCE M. CARLSON \\ Department of Anatomy, University of Michigan, Ann Arbor, Michigan 48109
}

\begin{abstract}
Patterns of activity of six dehydrogenase enzymes were studied histochemically in $\mathbf{4 2}$ free muscle grafts in the rat. Within hours, the surviving peripheral muscle fibers can be distinguished from the central ischemic muscle fibers. The surviving muscle fibers retain their characteristic pattern of staining throughout the post-transplantation period. The central ischemic muscle fibers stain abnormally and by five or six days they lose their enzymatic activity. The zone of regeneration, between the surviving and the ischemic muscle fibers, initially shows little dehydrogenase activity, but as the regenerating muscle fibers mature, they develop first a homogeneous staining pattern and, later, differences in staining intensity among different types of muscle fibers.
\end{abstract}

In a free muscle graft, the muscle is removed from its bed; all tendinous, vascular, and neural connections are severed, and the muscle is then replaced into its own bed or into another site. Although the tendons are resutured, no attempt is made to reanastomose blood vessels or nerves. Following transplantation, the muscle is characterized by a thin peripheral rim of surviving muscle fibers and a larger central area, in which ischemic muscle fibers are destroyed and replaced by newly regenerating muscle fibers. The destruction of old and the regeneration of new muscle fibers follows a centripetal gradient that is spatially and temporally correlated with the ingrowth of new blood vessels into the graft (Carlson et al., '79).

One of the major persisting questions in muscle grafting concerns the nature of the metabolic activities within the graft. Although pure biochemical studies allow the quantitation of total enzyme activity (Wagner et al., '77), they do not allow accurate localization of the metabolically active regions. Histochemical studies, although quantitatively less accurate, permit the precise localization of substrates or enzyme activity to specific areas within the graft. It is for this reason that we undertook a histochemical survey of dehydrogenase enzymes in early free muscle grafts.

\section{MATERIALS AND METHODS}

In 42 male Sprague-Dawley rats (150-200 g) one extensor digitorum longus (EDL) muscle was completely removed from the leg and then freely grafted back into its own bed. The contra- lateral muscle served as a normal control. At 2 and 4 hours and $1-10,15,16,20,26,30,35,36$, 38,42 , and 44 days after transplantation, grafted and control muscles were removed from the rats. The proximal half of each graft was frozen in a mixture of dry ice and isopentane and cross-sectioned at $7 \mu \mathrm{m}$ on a cryostat. The distal halves of the grafts were fixed in Bouin's solution, embedded in paraffin, and crosssectioned at $7 \mu \mathrm{m}$ for subsequent staining with Ehrlich's hematoxylin and eosin for morphological comparison with the histochemical preparations.

Histochemical activity of the following dehydrogenase enzymes was examined: lactate dehydrogenase (LDH), succinate dehydrogenase (SDH), malate dehydrogenase (MDH), hydroxybutyrate dehydrogenase $(\mathrm{BDH}), \alpha-$ glycerophosphate dehydrogenase ( $\alpha$-GPDH), and glucose-6-phosphate dehydrogenase (G6PDH). The procedure followed for demonstrating SDH activity was that of Nachlas et al. (57), with malonate controls. Pearse's ('72) standard method (pp. 1342-1343) with the nitro-blue tetrazolium salt was used for $\alpha$ GPDH and BDH. Activity of the soluble enzymes $\mathrm{LDH}, \mathrm{MDH}$, and G-6-PDH was also demonstrated by Pearse's standard method

Received February 2, 1979; accepted July 28, 1980.

Davindra K. Magon's present address is Department of Zoology, Kenyatta University College, Nairobi, Kenya.

Address reprint requests to Bruce M. Carlson, Dept. of Anatomy, Univ. of Michigan, Ann Arbor, MI 48109 
(composition of stock substrate solutions and incubation media given on p. 1344). Nitro-blue tetrazolium salt was again used in the precipitation reaction. Control for nonspecific staining was done by substrate deletion.

\section{RESULTS}

Untouched control muscles gave the expected histochemical reactions for all of the enzymes tested. An irregular "checkerboard" pattern of muscle fibers with low and high enzyme activity was seen in all of the control muscles (Fig. 1).

The postoperative course of a free muscle graft is complex, with varying numbers of zones of different types of developmental activity appearing at different stages (Carlson et al., '79; Hansen-Smith and Carlson, '79). Because of this and because within any given zone there is a remarkable similarity in the patterns of dehydrogenase activity, the histochemical results will be presented as patterns of activity within a graft at a particular stage.

The zonation pattern in a muscle graft varies continuously with time. Soon after grafting the muscle becomes subdivided into a thin (2-6 cells thick) peripheral zone of surviving muscle fibers. This layer does not change during the postoperative period except for the denervation atrophy which reduces the diameters of the individual muscle fibers during the first three weeks. The remainder of the graft is initially in a state of ischemic necrosis and remains so until macrophages, associated with the ingrowing blood vessels, begin to break down the ischemic muscle fibers. From about three to six days a wave of fragmentation extends more deeply toward the interior of the muscle until no original muscle fibers remain. About a day after the complete removal of old muscle cytoplasm, the original basal laminae of the fragmented muscle fibers become occupied by regenerating myotubes. The appearance of muscle fiber regenerations thus follows a parallel, but later, centripetal gradient like that of the fragmentation process.

As early as four hours after transplantation it is possible to distinguish between the narrow peripheral rim of surviving muscle fibers and the central zone of ischemic tissue. One of the most striking changes is an artifactual one in which, in a pure cross section of the muscle, the surviving muscle fibers $(\sim 5 \%)$ remain normally oriented all around the periphery in a thin layer, whereas the central ischemic muscle fibers $(\sim 95 \%)$ assume an oblique orientation to the cut surface (Fig. 2). The basis for this artifact is not known. For the first two days the graft can be roughly divided into an outer peripheral rim of surviving muscle fibers, which retain an almost normal histochemical staining pattern with differential staining of muscle fibers, and a central zone of ischemic muscle fibers, which still stain quite heavily and with a differential pattern but which show obvious morphological evidence of damage, such as coarser deposits of the reaction product.

By three days, macrophages coming in with the ingrowing vasculature have broken down the muscle fibers in the outer portions of the original ischemic zone, and the grafts contain three principal zones of histochemical activity (Fig. 3). The outer zone of surviving muscle fibers has changed little from previous days.

Fig. 1. Cross section through a control EDL muscle. Lactic dehydrogenase reaction. This section illustrates the typical "checkerboard" pattern of staining of a normal muscle. $155 \times$

Fig. 2. Cross section through a 4-hour graft. Reaction for $\alpha$-glycerophosphate activity. The surviving muscle fibers (top) stain normally, whereas the ischemic muscle fibers stain more coarsely. In cross sections through early grafts, the ischemic muscle fibers invariably become oriented somewhat obliquely to the plane of section. $155 \times$.

Fig. 3. Cross section through a 3-day graft. Reaction for succinic dehydrogenase activity. The peripheral surviving muscle fibers (P) retain differential staining patterns. The central ischemic muscle fibers (C) retain enzyme activity, although the normal staining pattern is severely disrupted. At this stage, the zone of degeneration and regeneration $(R)$ shows little enzymatic activity. $155 \times$

Fig. 4. Cross section through a 6-day graft. Reaction for lactate dehydrogenase activity. The peripheral surviving muscle fibers $(\mathrm{P})$ retain their activity, whereas the ischemic muscle fibers (inside dashed line) have lost their activity. Greater activity is seen in the regenerating muscle fibers $(R)$. $101 \times$. 

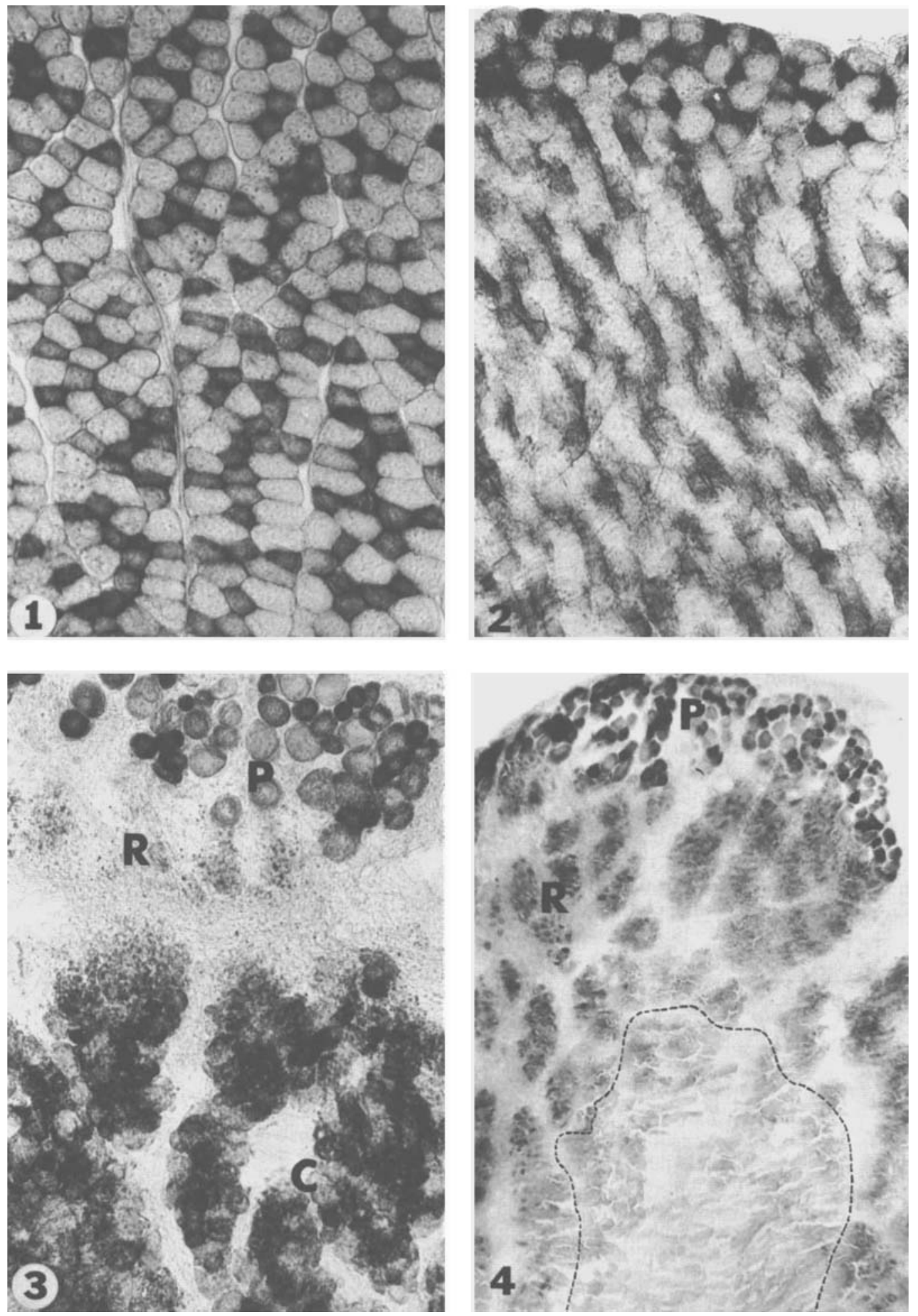
The central zone of ischemic original muscle fibers is smaller than before; these muscle fibers stain heavily, but the deposits are coarser than those of the surviving muscle fibers. Between these two zones is a zone with little dehydrogenase activity. This is the area in which the original muscle fibers have been completely broken down and the regenerating muscle fibers are still mostly in the myoblastic phase. From histochemical preparations one cannot determine whether the small punctate sites of activity in this zone are enzymatically active myoblasts or blood-borne phagocytic cells.

During the next couple of days the same zonal arrangement persists, with the central ischemic zone shrinking as the intermediate zone of degeneration and regeneration expands. By five or six days the remaining ischemic muscle fibers in the central region of the graft have lost virtually all dehydrogenase activity (Fig. 4). In contrast, the intermediate zone, which is now occupied by regenerating muscle fibers, demonstrates increasing enzymatic activity. Activity is most intense in the outer regions of this zone because there the regenerating muscle fibers are more mature. During this period, bundles of late myotubes could easily be identified on the basis of their LDH, BDH, and MDH activity. Neither earlier myotubes nor myoblasts could be distinctly identified on the basis of their dehydrogenase activity. G6PDH and SDH activities were also present, but that of SDH was noticeably less than that of the other dehydrogenases.

Early in the second week the original ischemic muscle fibers have all been removed and the graft consists of a peripheral rim of still identifiable, original muscle fibers surrounding a mass of young, cross-striated regen- erating muscle fibers (Fig. 5). The regenerating muscle fibers stain weakly, but distinctly (Fig. 6). There remains a slight gradient of staining intensity that parallels the gradient of maturation of the regenerating muscle fibers (Fig. 7), but among muscle fibers of a given stage of maturity the staining reactions are homogeneous. By the end of the second week it is sometimes difficult to differentiate between the more darkly staining surviving muscle fibers. Barely observable differences in staining reactions can be seen among some regenerating muscle fibers.

By 16 days the more mature regenerating muscle fibers begin to show slight differentiation of fiber types, and in 20- and 26-day grafts it is not possible to distinguish between the surviving muscle fibers and the outermost regenerating muscle fibers, which have differentiated into readily recognizable muscle fiber types. Further inward, the regenerating muscle fibers still retain fairly homogeneous and intermediate staining characteristics. Older grafts consist principally of histochemically differentiated muscle fibers. Although occasionally traces of developmental staining gradients persist, the major differences in staining reactions tend to be interfascicular (Fig. 8). As is already well established for ATPase staining (Carlson and Gutmann, '75), fiber type grouping is seen with all of the dehydrogenase enzymes tested in mature grafts. Occasional clusters of atrophied muscle fibers are typically seen among the maturing muscle fibers of older grafts.

\section{DISCUSSION}

The histochemical activity of the dehydrogenase enzymes that were tested was closely tied to the histological reactions of the muscle

Fig. 5. Cross section through an 8-day graft. Reaction for 3-hydroxybutyrate dehydrogenase activity. The peripheral, still-denervated muscle fibers (P) continue to show differential staining, whereas the regenerating muscle fibers $(R)$, which now occupy the entire center of the graft, stain lightly and homogeneously. $155 \times$.

Fig. 6. Cross section through the central area of an 8-day muscle graft. Lactic dehydrogenase reaction. All of the muscle fibers seen in this figure are regenerating and are lightly and homogeneously stained. The light spots in many of the muscle fibers are negative images of the nuclei. $155 \times$.

Fig. 7. Cross section through a 9-day graft. Surviving muscle fibers (on top) are still relatively large in diameter and retain some differentiated staining characteristics. In this graft a gradient of maturity of the regenerating muscle fibers from relatively large ones near the periphery of the graft to smaller, less advanced ones in the center of the graft (bottom). Succinic dehydrogenase. $155 \times$.

Fig. 8. Cross section through 35-day muscle graft, showing differential staining of regenerating muscle fibers for 3-hydroxybutyrate activity. The grouping of muscle fibers with similar staining reactions is evident. $155 \times$. 

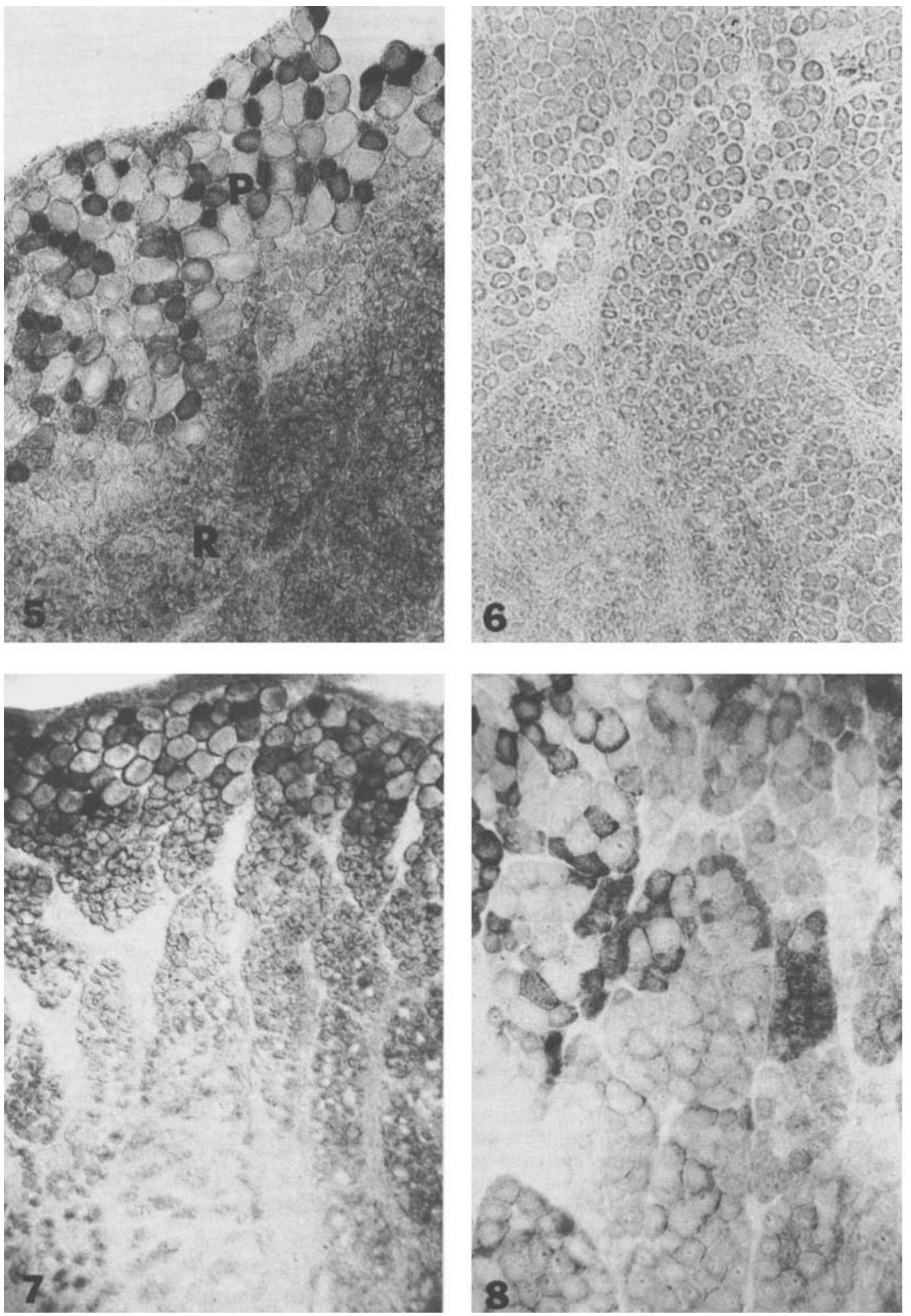
TABLE 1. Summary of histochemical studies of free muscle (EDL) grafts during critical postoperative periods ${ }^{1}$

\footnotetext{
4 hours Differences apparent between surviving peripheral and central ischemic muscle fibers-dehydrogenase enzymes.

1-2 days Surviving muscle fibers: Retain activity in checkerboard pattern-dehydrogenases, ATPase, phosphorylase, glycogen.

Central ischemic muscle fíbers: Dehydrogenase and ATPase activity present, but morphology altered. No phosphorylase and minimal glycogen.

3- days Surviving muscle fibers: Glycogen and activity of dehydrogenases, ATPase, phosphorylase like before, lipid droplets present.

Central ischemic fibers: Activity of remaining enzymes diminishes to nearly zero.

Zone of degeneration and regeneration: Minimal activity in areas of degeneration; areas of early regeneration, muscle stains faintly for dehydrogenase-LDH and BDH stronger than others. Phosphorylase and glycogen absent. ATPase, minimal activity in older fibers.

7-15 days Surviving muscle fibers: Lipid droplets gone; dehydrogenase and ATPase activity like before. Phosphorylase and glycogen present.

Regenerating muscle fibers: No phosphorylase, minimal glycogen, uniform dark ATPase, activity of all dehydrogenases seen-LDH and $\mathrm{BDH}$ show stronger centripetal gradient and G6PDH a weaker gradient than $\mathrm{SDH}, \mathrm{MDH}$, and $\alpha-\mathrm{GPDH}$.

15-30 days Distinetion between surviving and most mature regenerating muscle no longer possible with dehydrogenase enzymes - differential staining of muscle fibers begins; ATPase uniformly dark in regenerating muscle fibers. Some glycogen and phosphorylase.

$30+$ days Fiber type differentiation with ATPase as well as dehydrogenases. Phosphorylase returns-type grouping seen. By 45 days developmental gradients in graft are gone.

'Data on ATPase and phosphorylase from Carlson and Gutmann ('75), E/M of lipid from Hansen-Smith and Carlson ('79), histochemistry of lipid and glycogen from Magon, Basson and Carlson (unpublished).
}

fibers within the grafts. Table 1 summarizes, according to postoperative periods, the data obtained in this study along with information from previously published works. The peripheral, surviving muscle fibers apparently retain their viability by diffusion mechanisms and retain a relatively normal staining pattern throughout the postoperative period despite being nerveless for 2-3 weeks after grafting. Although the ischemic muscle fibers of the grafts lose virtually all phosphorylase activity within hours (Carlson and Gutmann, '75; Wagner et al., '77), the staining for dehydrogenases remains prominent for several days after grafting. By five or six days, however, almost all activity in the ischemic muscle fibers is lost. This observation parallels that of Snow ('73) on minced muscle regeneration, and some of the characteristics of the staining reactions are undoubtedly due to damage to mitochondria and other intracellular structures (Hansen-Smith and Carlson, '79). This probably accounts for some of the apparent contradictions between histochemical and biochemical studies on degenerating and regenerating muscle (Snow, '73; Wagner et al., '77).

There was a close similarity in the reactions of all of the dehydrogenases tested. Although there were minor quantitative differences in the intensity of the staining reactions and occasionally in the clarity with which the gradient of maturity of regenerating muscle fibers could be demonstrated, the overall qualitative patterns in relation to space and time were virtually identical. One difference between the present results and previous reports (Snow, '73) is a somewhat higher activity of glucose-6phosphate dehydrogenase in both normal muscle and in mature regenerating muscle in this study.

Histochemical staining permits a much more accurate differentiation between surviving and regenerating muscle fibers in regenerates between one and two weeks of age than does histological staining. The oldest regenerating muscle fibers are often as large as the smaller surviving fibers, which are somewhat atrophied because of having been denervated since the time of grafting, but their staining reactions are still immature.

\section{ACKNOWLEDGMENT}

This work was supported by grants from the Muscular Dystrophy Association and $\mathrm{NIH}$ (NS-13116).

\section{LTTERATURE CITED}

Carlson, B.M., and E. Gutmann (1975) Regeneration in free 
grafts of normal and denervated muscles in the rat: Morphology and histochemistry. Anat. Rec., 183:47-61.

Carlson, B.M., F.M. Hansen-Smith, and D.K. Magon (1979)

The life history of a free muscle graft. In "Muscle Regeneration." A. Mauro et al., (eds.). Raven Press, New York, pp. 493-507.

Hansen-Smith, F.M., and B.M. Carlson (1979) Cellular responses to free grafting of the extensor digitorum longus muscle of the rat. J. Neurol. Sci., 41:149-173.

Nachlas, M.M., K.C. Tsou, E. Souza, C.S. Cheng, and A.M. Seligman (1957) Cytochemical demonstration of succinic dehydrogenase by the use of a new p-nitrophenyl substituted ditetrazole. J. Histochem. Cytochem., 5:420-436.

Pearse, A.G.E. (1972) Histochemistry, Ed. 2, Vol. 2. Churchill Livingstone, Edinburgh.

Snow, M.H. (1973) Metabolic activity during the degenerative and early regenerative stages of minced skeletal muscle. Anat. Rec., 176: 185-204.

Wagner, K.R., B.M. Carlson, and S.R. Max (1977) Developmental patterns of glycolytic enzymes in regenerating skeletal muscle after autogenous free grafting. J. Neurol. Sci., 34:373-390 